\title{
GESTIÓN TRADICIONAL DE LA ESCORRENTÍA DE TORMENTAS PARA RIEGO DE PRADOS EN MONTEAGUDO DEL CASTILLO (TERUEL)
}

\author{
Alejandro J. Pérez Cueva \\ alejandro.perez@uv.es \\ Ghaleb Fansa Saleh \\ ghaleb.fansa@uv.es \\ Departamento de Geografía, Universidad de Valencia, \\ Av. Blasco Ibáñez, 28, 46010 Valencia
}

\begin{abstract}
Resumen: Monteagudo del Castillo (Teruel) está cerca del polo de máxima tormentosidad violenta de la Península Ibérica. El aporte pluviométrico de estas tormentas es capaz de invertir el régimen estacional habitual del clima mediterráneo, con el verano como estación más lluviosa. Aquí se estudia la gestión tradicional de la arroyada en dos cuencas del término municipal, desde la doble perspectiva riesgo/recurso: control de la erosión y aprovechamiento de la arroyada para regar prados. Para gestionar la arroyada concentrada de las tormentas se ha modificado la red de drenaje mediante acequias recolectoras y/o evacuadoras y la rectificación de la red hidrográfica natural. Las acequias de derivación permiten la existencia de prados para ganado bovino con precipitaciones alrededor de $430 \mathrm{~mm} / \mathrm{año}$. Se reconstruye la red de drenaje mediante trabajo de campo, fotointerpretación y datos LIDAR, para conceptualizar los tipos de cauce, interpretar la red antrópica y poder determinar las estrategias de gestión.
\end{abstract}

Palabras clave: Tormentas, arroyada concentrada, water harvesting, Alto Alfambra, red de drenaje antrópica. 


\title{
Traditional storm runoff management for irrigating meadows in Monteagudo del Castillo (Teruel)
}

\begin{abstract}
Monteagudo del Castillo (Teruel) is near the pole of maximum violent storms of the Iberian Peninsula. The pluviometric contribution of these storms is capable of reversing the usual seasonal regime of the Mediterranean climate, with summer as the rainiest season. Here the traditional management of the stream in two basins of the municipal area is studied, from the double perspective risk/resource: control of erosion and use of the stream to irrigate meadows. To manage the concentrated stream of the storms, the drainage network has been modified through collecting and/or evacuating channels and the rectification of the natural hydrographic network. The derivation channels allow the existence of meadows for cattle with rainfall of around $430 \mathrm{~mm} /$ year. The drainage network is reconstructed through field work, photointerpretation and LIDAR data, to conceptualize the channel types, interpret the anthropic network and determine management strategies.
\end{abstract}

Keywords: Thunderstorms, concentrated runoff, water harvesting, Alto Alfambra, anthropic drainage network.

\section{Introducción}

La gestión de escorrentías en laderas como recurso hídrico presenta dos tipos de elementos comunes, los de captación de agua en las laderas y los de distribución posterior en los campos de cultivo en secano (Beckers, Berking y Schütt, 2013). Dentro del espacio cultivado se produce un mayor o menor aprovechamiento, según las características climáticas, mediante variadas soluciones. Pero suele ser habitual que exista una componente de riesgo más o menos importante, que coexiste con el aprovechamiento del agua como recurso. Este riesgo depende, sobre todo, del carácter torrencial de las lluvias. La adaptación al riesgo determina otro tipo de soluciones.

Tanto el grado de riesgo/recurso que tienen que solucionar/aprovechar los sistemas, como las soluciones empleadas y su grado de aplicación, dependen de dos variables climáticas, la pluviosidad general y la torrencialidad de las lluvias, muy variables en el clima mediterráneo. De ello se deduce que, más que de tipos de sistemas en concreto, estamos delante de casos de "diseño a medida", con una casuística muy grande. La complejidad de las soluciones adoptadas depende del grado de organización posible, desde sistemas particulares hasta organización de espacios mucho más amplios con muchos propietarios (como el sistema de meskat en los alrededores de Sousse) (El Amami, 1983). 
En el SE español son numerosos los casos de derivación de aguas de escorrentía, aunque la mayor parte no han merecido un estudio detallado, excepto quizá el de las boqueras (Hernández y Morales, 2013). Muchos de ellos son soluciones simples que obedecen al interés de reducir el riesgo de erosión o de aprovechar mejor los fondos de valle. Muchas de estas actuaciones son simples correcciones del trazado de los canales naturales.

El objetivo de este trabajo es estudiar la gestión tradicional del agua como riesgo/recurso en Monteagudo del Castillo (Teruel). Se trata de un ejemplo de gestión de la escorrentía de tormentas para regar prados, extensible a otros lugares de la cuenca del Alto Alfambra. Es un espacio reordenado de mayor extensión que lo habitual (gran parte de pequeñas cuencas), que pertenece a un ámbito ombrotérmico cercano a la transición entre semiárido y subhúmedo, sobre todo por las bajas temperaturas estivales (Cuadrat, Saz y Vicente, 2007). En las diferentes estrategias y soluciones de gestión del agua predomina la faceta de riesgo de las lluvias, más que su valor como recurso. Los objetivos concretos de este trabajo son reconstruir y conceptualizar la red de drenaje, tanto la natural como la antropizada, y evaluar la gestión de la arroyada como riesgo y/o recurso.

\section{1. Área de estudio}

Los barrancos de San Cristóbal y de La Cañadilla son dos pequeñas cuencas de 5,19 $\mathrm{km}^{2}$ y $0,90 \mathrm{~km}^{2}$ de extensión, situadas en las cercanías de Monteagudo del Castillo (Teruel) (ver figura 1). Son dos afluentes del Río Seco, curso fluvial que aporta sus aguas al Alfambra y Turia.

\subsection{Geología}

Monteagudo se localiza en el tránsito entre la Depresión de El Pobo y la sierra de Gúdar, a caballo entre las cuencas del Mijares y Alfambra. En conjunto se pueden observar dos grandes sectores geológicos: la cubeta sinclinal del Pobo y el anticlinal de Alcalá. El sector estudiado pertenece totalmente a esta cubeta sinclinal, que enlaza dos estructuras elevadas, el anticlinal de Alcalá y el de Hoyalta-Castelfrío. En ella afloran casi exclusivamente las calizas, margas y areniscas calcáreas del Portlandiense, en disposición subhorizontal. También afloran conglomerados con intercalaciones de arcillas del Mioceno, que pueden observarse en las lomas de La Sabina y de Los Arcos, en Las Cabezadas, y también en el techo del cerro del Castillo. Este cerro-testigo, coronado de Mioceno, es un buen indicador de hasta qué altura llegó el relleno de materiales detríticos. Posteriormente, durante el Plioceno y el Cuaternario, el encajamiento de la red fluvial vació casi totalmente estos materiales e hizo aflorar el Portlandiense. 


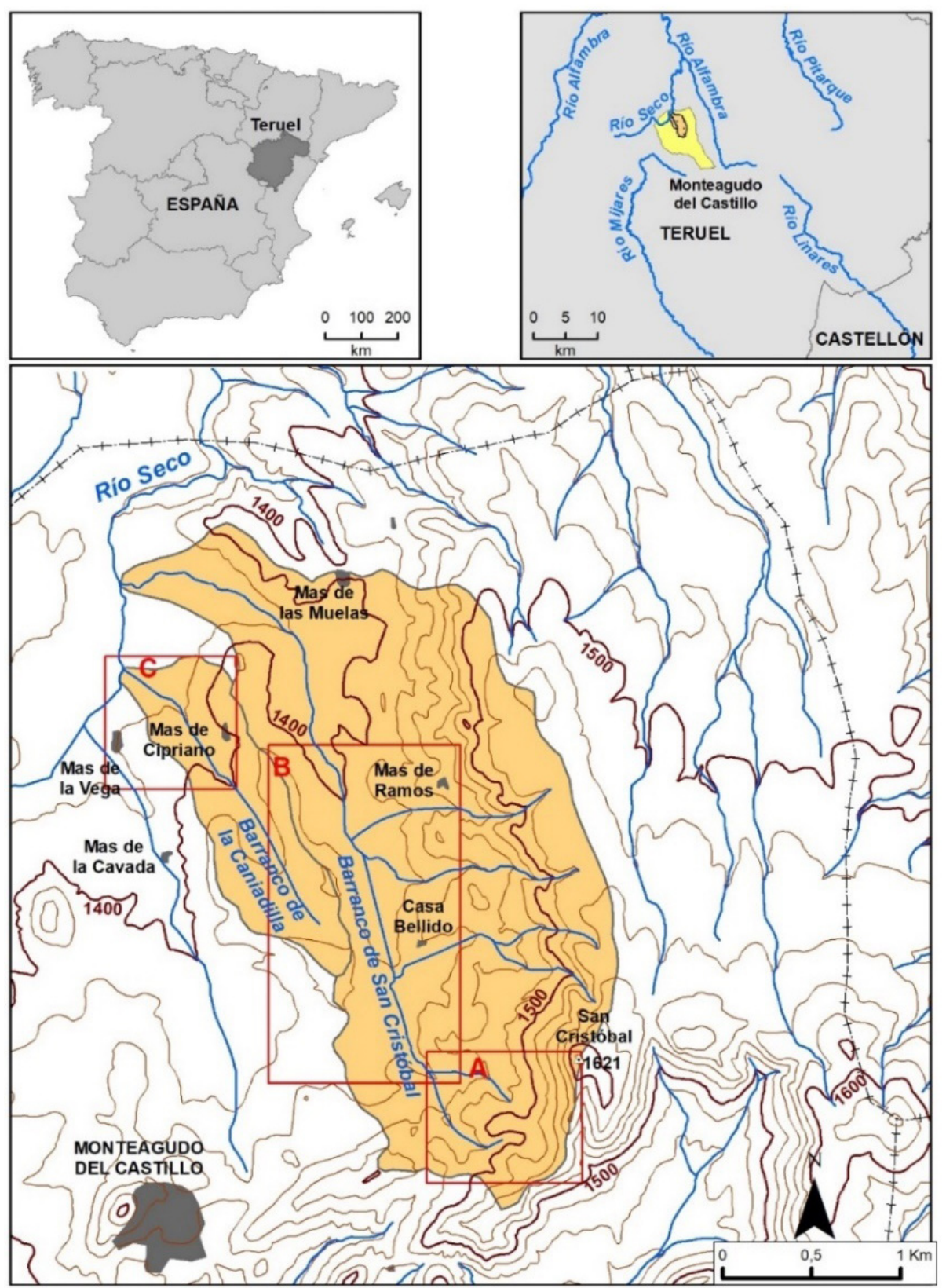

Figura 1. Localización de la zona de estudio de Monteagudo del Castillo (Teruel). Fuente: Elaboración propia. 

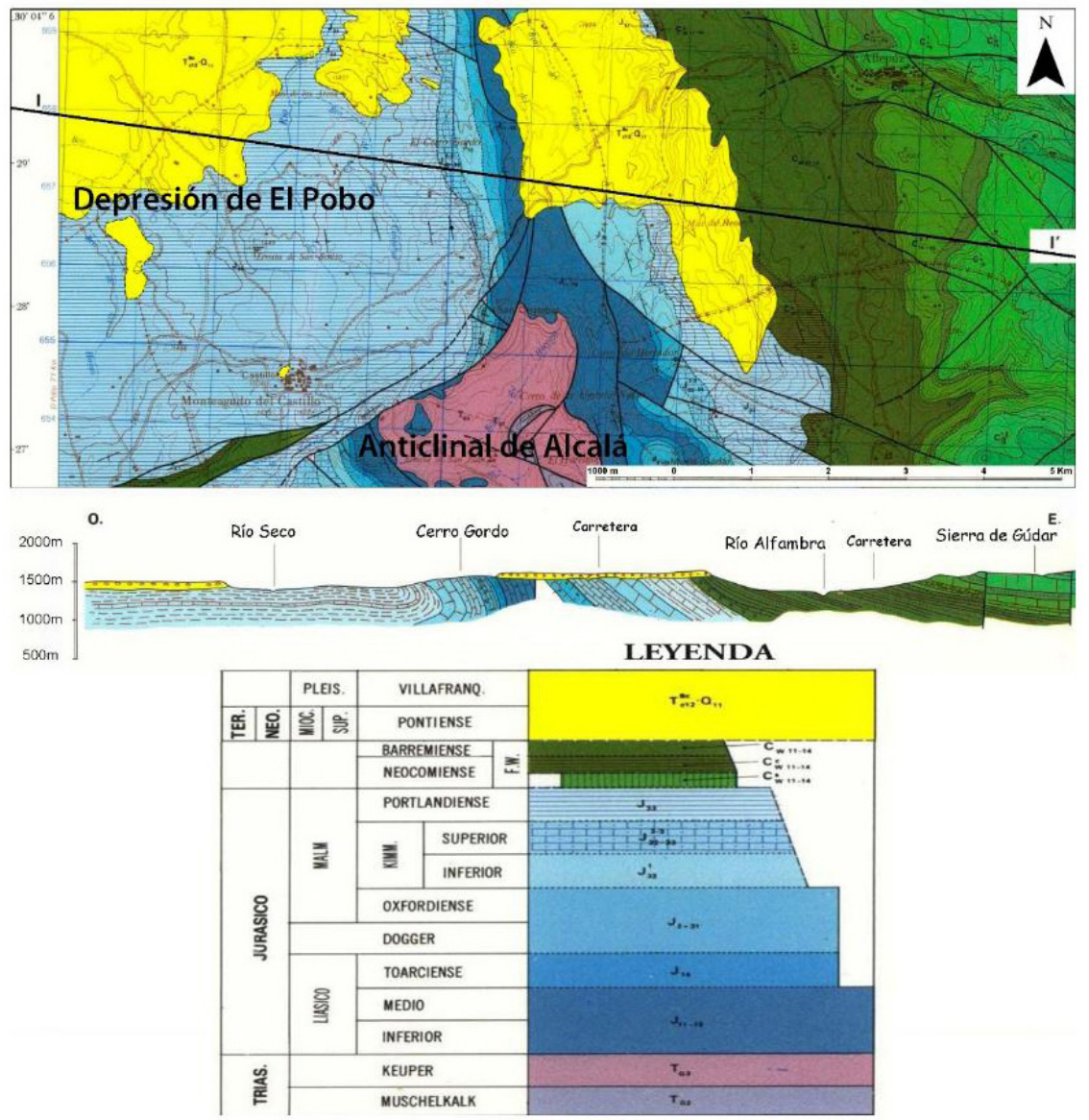

Figura 2. Mapa y corte geológico de los alrededores de Monteagudo del Castillo, mostrando la Depresión de El Pobo y el extremo norte del anticlinal de Alcalá.

Fuente: Penalba y Pérez Cueva (2013), adaptado de IGME (1981).

La zona ha sido estudiada por Lozano (1983) y Pailhé (1984) desde un punto de vista geomorfológico, y por Pailhé (1971) desde el geológico. En estos estudios se muestra la depresión del Pobo a la vez como una cubeta tectónica y una depresión de vaciado. Más recientemente, su relleno neógeno ha sido estudiado por Simón-Porcar (2017).

Los materiales del Portlandiense, presentes en casi la totalidad de las dos cuencas, están constituidos por una serie carbonatada alternante de calizas, areniscas calcáreas, margas e incluso algunos niveles de arcillas. Las margas portlandienses son muy impermeables y erosionables, por lo que la torrencialidad de las lluvias, en particular las de las tormentas estivales, genera fácilmente contextos de riesgo. 


\subsection{Climatología}

El clima de Monteagudo del Castillo pertenece al tipo submediterráneo continental frío, según el Atlas Climático de Aragón, (Cuadrat, Saz y Vicente, 2007), común a casi toda la provincia de Teruel. Se caracteriza por unas temperaturas mínimas muy bajas, unas máximas algo moderadas para un clima continental, por efecto de la altitud, y unas precipitaciones generalmente escasas. En lugares protegidos con mínimos pluviométricos, como las depresiones del Alfambra y del Jiloca (o la misma Depresión del Pobo) este clima llega a ser estepario. En lugares más montañosos, como la Sierra de Gúdar, la precipitación aumenta ligeramente, lo que unido al descenso térmico es suficiente para alcanzar unas condiciones casi subhúmedas. El contexto climático concreto de la zona de estudio es el de una rápida transición entre ambientes subáridos y subhúmedos, aunque más cercano al primero.

\subsubsection{El régimen térmico}

La altitud y la ubicación de la zona de estudio son los principales condicionantes de sus rasgos climáticos. Las temperaturas no sobrepasan los $10^{\circ} \mathrm{C}$ de media anual. La media del mes más frío (enero) apenas supera $1^{\circ} \mathrm{C}$, mientras que la media del mes más cálido (julio) se sitúa sobre $19^{\circ} \mathrm{C}$. Esto da lugar a una amplitud media anual de unos $18^{\circ} \mathrm{C}$, propia de un régimen térmico mediterráneo continental. Esta continentalidad se basa más en las rigurosas temperaturas invernales que en las temperaturas estivales, muy moderadas por la altitud.

\subsubsection{Las precipitaciones}

Se reparten de un modo bastante equilibrado a lo largo del año. En invierno es cuando se registra la estación más seca, con precipitación en torno a $70 \mathrm{~mm}$. La primavera también es bastante seca, con unos 100 $\mathrm{mm}$. El verano es la estación ligeramente más lluviosa, con cantidades cercanas a $130 \mathrm{~mm}$. Recoge la pluviosidad tormentosa de junio y sobre todo la de agosto, típica de final del verano, pero que se extiende a veces a todos los meses cálidos. El otoño registra unas precipitaciones comparables al verano, aunque ligeramente inferiores, sobre $120-130 \mathrm{~mm}$, al estar la zona resguardada de la influencia mediterránea.

La localización concreta del término lo protege de casi todas las situaciones atmosféricas que producen precipitación: de los temporales de Levante (por el macizo de Gúdar), de los del SW (por el eje Javalambre-Montes Universales) y de los del N y NW (sistemas orográficos del norte peninsular). Aun así, los temporales de levante debilitados son responsables de un $40 \%$ de las avenidas de la cuenca del Alfambra. Sin 
embargo, el mecanismo de las precipitaciones convectivas es el más eficiente, pues, aunque funciona sólo en los meses más cálidos, llega a producir el $30 \%$ de las avenidas de la cuenca (Morell-Cuevas, 2001). A pesar de ello, el estar situados en tránsito entre la Depresión del Alfambra y el macizo de Gúdar hace que las situaciones atmosféricas lluviosas con componente $\mathrm{W}$ recuperen en parte su pluviosidad por efecto orográfico. En total, se alcanzan unos $435 \mathrm{~mm}$ de media anual (figura 3).
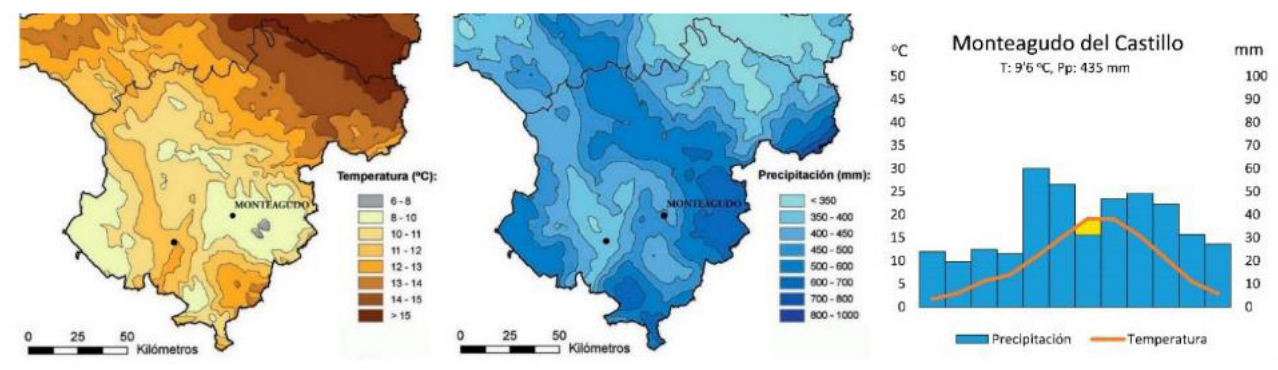

Figura 3. Distribución espacial de las temperaturas medias anuales y las precipitaciones totales medias anuales de la provincia de Teruel y climograma de Monteagudo.

Fuente: Atlas Climático de Aragón (Cuadrat, Saz y Vicente, 2007) y elaboración propia.

Hay que señalar que el macizo de Gúdar es, junto al Pirineo Oriental, uno de los polos de máxima tormentosidad de la Península Ibérica, y el que registra mayor número de tormentas violentas (bailstorms) (figura 4).

\section{Número de días al año con tormentas fuertes}

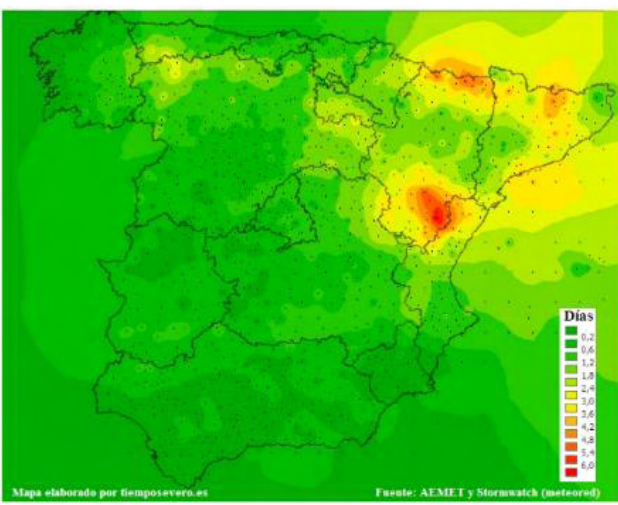

\section{Número de días al año con tormentas}

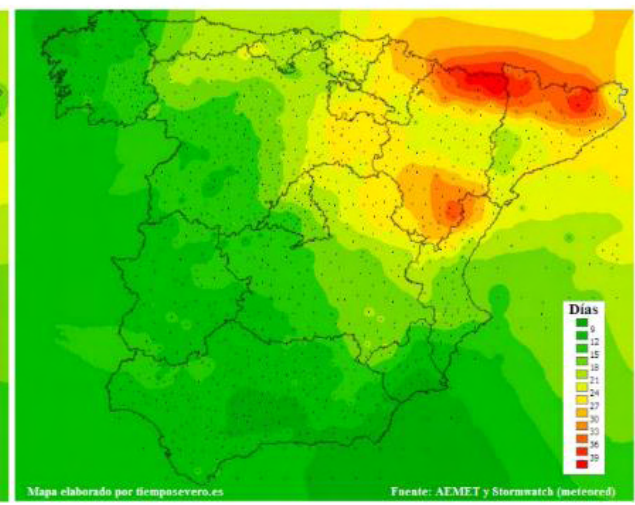

Figura 4. Tormentosidad de la Península Ibérica. El macizo de Gúdar es el principal polo peninsular de tormentas violentas (hailstorms) de la península.

Fuente: González Márquez (2006), a partir de Stormwatch, Meteored. 


\subsubsection{La evapotranspiración y el balance hídrico}

Las bajas temperaturas (figura 3) son la causa de que la evapotranspiración potencial media anual se sitúe en torno a $900 \mathrm{~mm}$, muy limitada en invierno, inicio de la primavera y final de otoño. La evapotranspiración potencial se basa pues en las temperaturas del semestre estival. Como se trata además del momento más lluvioso del año, ello incrementa la evapotranspiración real y acentúa las características semiáridas de la zona, que no llegan a ser excesivas, a causa de las moderadas las temperaturas estivales.

El carácter tormentoso de las lluvias, con cantidades que pueden superar los $50 \mathrm{~mm} /$ día con cierta facilidad, junto con ocasionales nevadas copiosas invernales, son los principales procesos de recarga hídrica. Son recargas muy eficientes, según muestran las numerosas surgencias del término municipal (Penalba y Pérez-Cueva, 2013) en todos los contextos hidrogeológicos posibles (Penalba, Pérez-Cueva y Fansa, 2012 y Villarroya, Pérez-Cueva, Fansa, y Penalba, 2014).

En Monteagudo, pues, se reúnen un conjunto de factores que sitúan la gestión tradicional del agua a caballo entre el riesgo (en mayor medida) y el recurso:

- La presencia de las margas del Portlandiense.

- Unas precipitaciones escasas y que caen fundamentalmente en los meses de más calor.

- Una torrencialidad notable, de la mano de las tormentas convectivas, que pueden superar más de $100 \mathrm{~mm}$ /día con periodos de recurrencia relativamente cortos (10-20 años).

Aparte de estos condicionantes del medio físico, también es importante observar que la dedicación agropecuaria tradicional y actual está basada en el cultivo del cereal y en prados para el ganado (principalmente el bovino). Las aguas de avenida de las tormentas estivales, sobre todo las de julio y agosto, son un riesgo para el cereal, pero un importante recurso para mantener verdes los prados en el periodo de máximo déficit hídrico.

\section{Metodología}

Se ha realizado fundamentalmente trabajo de campo y entrevistas. El trabajo de campo ha consistido en el reconocimiento exhaustivo de todos los canales funcionales de la red de los barrancos de San Cristóbal y la Cañadilla. A la vez que se constataba la funcionalidad, se han tipificado los tramos en "natural" y "antropizado", y dentro de estos últimos se han establecido diferentes tipos: acequias según su función principal (de recolección, de evacuación y de derivación), tramos de cauces "rectificados" (con trazados rectos nuevos) y tramos "lateralizados" (desplazados al lateral). 
A la vez, se han observado y clasificado otros elementos de control de la erosión o de aprovechamiento del agua, y se ha entrevistado a vecinos de Monteagudo para confirmar las identificaciones dudosas (en especial la de los prados).

\subsection{Reconstrucción de la red funcional: conceptos}

En esa tarea se han conceptualizado las funciones de cada uno de los tramos de cauce que constituyen la red. En total, se han utilizado los siguientes conceptos:

1. Red natural: Cuando la red funcional coincide con la reconstrucción topográfica de la red "natural". Se entiende en tal caso que no existe una corrección de cauces suficientemente significativa. Suele presentar un característico trazado irregular y está sistemáticamente en la posición topográfica más baja.

2. Flujo en bancales: Cuando la red funcional desaparece porque los bancales rompen su conectividad natural o alteran la dirección de flujo. En ocasiones puede coincidir la dirección del flujo topográfico natural con el camino que sigue el agua entre los bancales, pero normalmente estos están diseñados para dispersar o concentrar el flujo según lo que más interese al agricultor. El criterio adoptado es que, si el flujo no muestra un cauce aparente, pertenece a este tipo.

3. Cauces enderezados: Se trata de flujos canalizados con tramos rectos situados en fondo de valle. Son elementos de la red funcional que aumentan la conectividad, por lo que su papel fundamental es acelerar la evacuación de agua.

4. Cauces laterales: Es otro tipo de corrección de cauces, consistente en desplazarlos claramente a un lateral del bancal. Se utiliza casi exclusivamente en vaguadas estrechas, para aprovechar todo el fondo como labor.

5. Acequias recolectoras: Las acequias, a diferencia de los cauces, son elementos de la red funcional que no existían previamente. No se trata de modificaciones del drenaje, sino elementos totalmente nuevos y con una función definida. Las acequias recolectoras tienen la función de interceptar el drenaje, concentrarlo y prepararlo para su evacuación, para que no llegue a los campos de cultivo o se acumule en exceso. Su objetivo principal es controlar la erosión.

6. Acequias evacuadoras: Tienen la función de drenar hacia la red principal la arroyada acumulada en los campos de cultivo o la concentrada ya por las acequias recolectoras. Aumentan la conectividad hidrológica en contextos en que los bancales la interrumpen. Como sólo funcionan en casos de lluvias intensas, protegen de la erosión las zonas abancaladas cuando hay un exceso de escorrentía. Tienen el inconveniente de que, si están mal diseñadas, pueden generar cárcavas.

7. Acequias de derivación: Tienen el papel de derivar el agua ya concentrada hacia lugares en que pueda aprovecharse (balsas, derrames, riego de prados...). 
Normalmente las acequias suelen tener más de una función, pero siempre hay una principal. Según el contexto en el que estén, puede desempeñar una segunda función "en pasivo", es decir, por estar allí. En tales casos, se han cartografiado según su función principal o "activa".

En la zona, hay una modalidad de acequias denominada localmente "enjuagadores" (Villarroya et al., 2014), que sirve para drenar zonas excesivamente húmedas. Serían un subtipo de acequia evacuadora, pero sin la función de evitar la erosión.

\subsection{Reconstrucción de la red "topográfica"}

La reconstrucción de la red funcional se ha complementado con el trazado de la red "natural". Se entiende como tal, no solo la que se observa como funcional no antropizada, sino también la que existiría en muchos lugares (sobre todo en las vaguadas) si no hubiese campos labrados: cuando los cauces concentran poca agua, el laboreo los hace desaparecer y los bancales ocupan el eje de las vaguadas, donde se concentra la humedad.

Para reconstruir la red "natural" se ha utilizado como fuente los datos LIDAR facilitados por el IGN (de fecha 2014). A partir de ellos se elaborado un modelo digital del terreno con una resolución de $1 \mathrm{~m}$. Se analiza la procedencia del agua en cada celda para obtener la red fluvial "topográfica", o recorrido que hace el agua con la topografía actual.

Los pasos seguidos son:

1. Rellenar las celdas ciegas (endorreicas) o corregir las imperfecciones en el modelo digital de elevaciones (Fill sinks), de manera que dichas celdas imperfectas tengan una altura igual a la mínima altura de las ocho celdas que la rodean.

2. Definir la dirección del flujo (Flow direction) buscando el camino descendente de una celda a otra.

3. Crear el raster de acumulación de flujo en cada celda (Flow accumulation). Se determina el número de celdas de aguas arriba que vierten sobre cada una de ellas.

4. Clasificar las celdas con acumulación de flujo superior a un umbral como celdas pertenecientes a la red hidrográfica (Stream definition), considerando como red hidrográfica todas las celdas que reciben un mínimo de superficie de captación. En este caso se ha establecido como umbral 20.000 celdas $\left(20.000 \mathrm{~m}^{2}\right)$ para empezar a considerar la celda como canal.

5. Crear un shapefile de drenajes (Stream feature) para facilitar los cálculos y las tareas de representación cartográfica. 


\section{Resultados: La antropización del drenaje en los barrancos de San Cristóbal y La Cañadilla}

Este conjunto de cuencas, y otras adyacentes, presentan diversas soluciones para corregir los dos problemas básicos señalados: controlar la erosión y aprovechar las aguas de avenida lo máximo posible.

Entre las medidas para corregir la erosión tenemos fundamentalmente tres: abancalamiento de laderas, corrección de cauces y establecimiento de acequias de drenaje. Entre las soluciones de aprovechamiento de las aguas turbias, la medida fundamental es la de derrames en prados, y puntualmente las balsas recargadas con escorrentías.

\subsection{El control de la erosión}

\subsubsection{Sistemas de bancales}

Se disponen en los lugares de mayor pendiente de las cuencas. Son el sistema de defensa casi exclusivo en la cabecera del Barranco de San Cristóbal (figura 5). En otros lugares son menos abundantes, pero dada la topografía ondulada de todo el sector pueden estar presentes en cualquier lugar (ej. figura 8 y figura 9).

Las litologías que afloran en las cuencas suministran material para la construcción de paredes de piedra seca. Sin embargo, a pesar de esta disponibilidad, no siempre se utilizan, y bastantes de los bancales son taludes fijados con vegetación espontánea o matorral (figura 6). Sólo en lugares de pendiente elevada se utiliza sistemáticamente la piedra. También puede darse un caso mixto de talud reforzado con piedras en la parte superior.

\subsubsection{Acequias de derivación}

La presencia de litologías blandas y la elevada torrencialidad de las lluvias ocasionan que cualquier pequeña cuenca, vaguada o ladera pueda producir escorrentías capaces de generar erosión en los campos de cultivo. El elemento crítico es la rotura de conectividad hidrológica que, en sectores semiáridos y áridos, generan los bancales (Bracken y Croke, 2007; Meerkerk, van Wesemael y Bellin, 2009), pues al ser la superficie cultivada necesitan captar el máximo de agua (Arnáez, Lana, Lasanta, Ruiz y Castroviejo, 2015). En Monteagudo, sin embargo, en casos de lluvias fuertes es necesario que funcione la conectividad hidrológica y que ésta sea compatible con la existencia de bancales. Esta es la función que persiguen las acequias de recolección y derivación de aguas de escorrentía. 


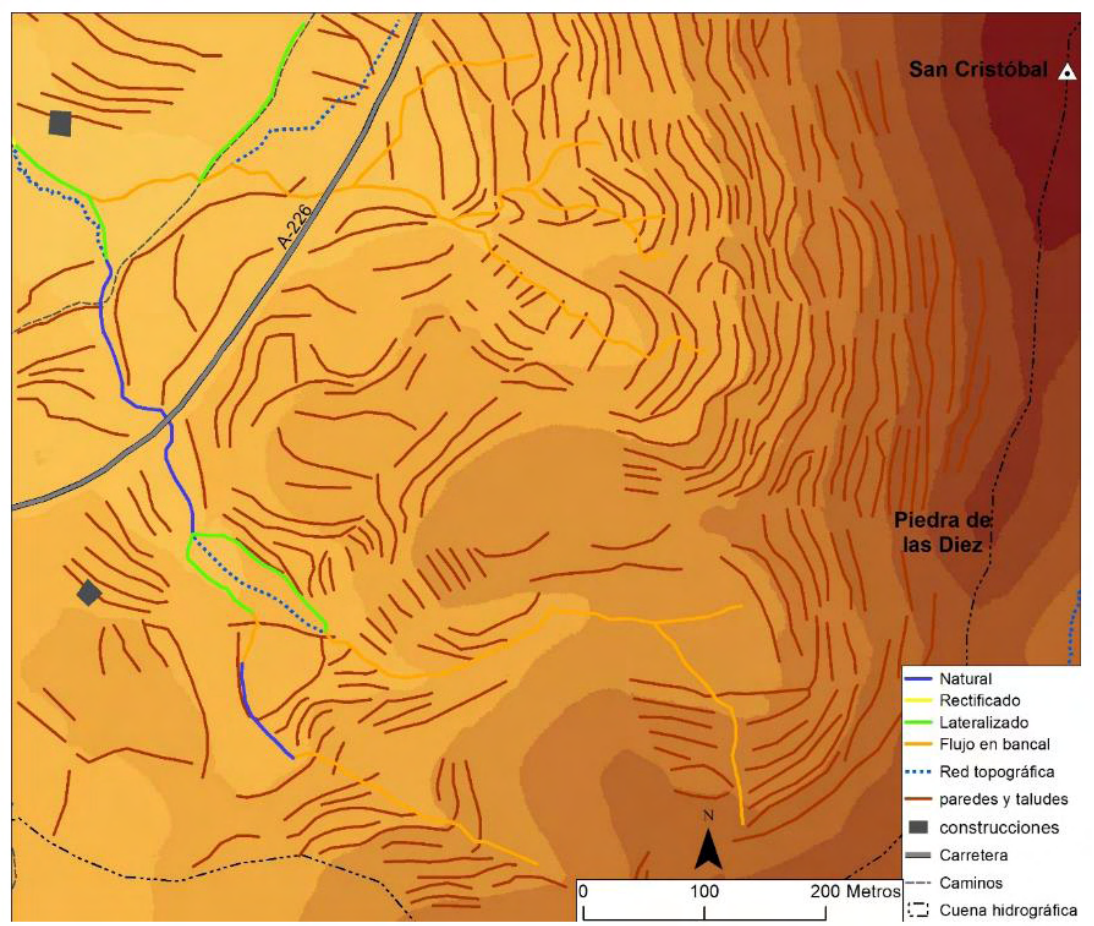

Figura 5. Red de drenaje, elementos de defensa contra la erosión y aprovechamiento de aguas de escorrentía en la cabecera del Bco. de San Cristóbal.

Fuente: Elaboración propia.

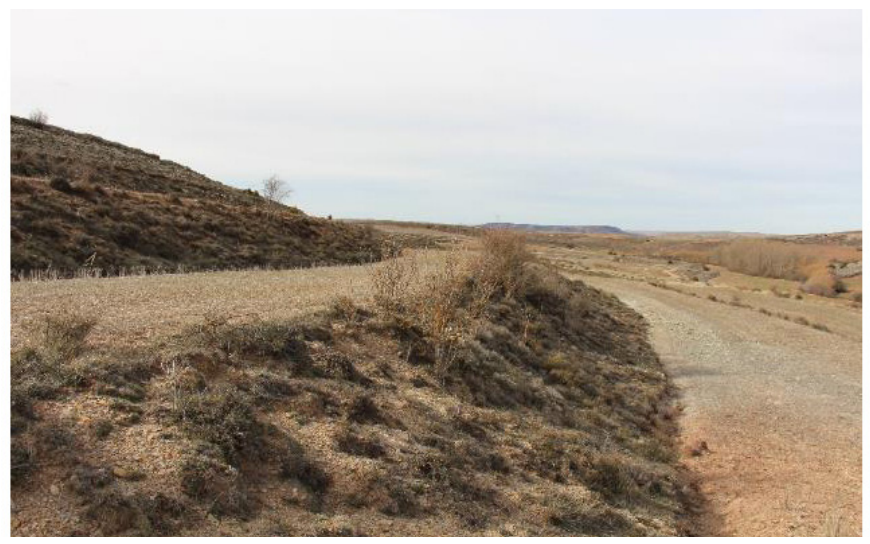

Figura 6. Ejemplo de bancales con talud vegetado.

Fotografía de los Autores. 
Por esta razón, un lugar característico de ubicación de acequias de recolección es en la base de las laderas, justo antes del bancal más alto (figura 7A), que conectan con otras de evacuación hasta el barranco principal (figura 7B).

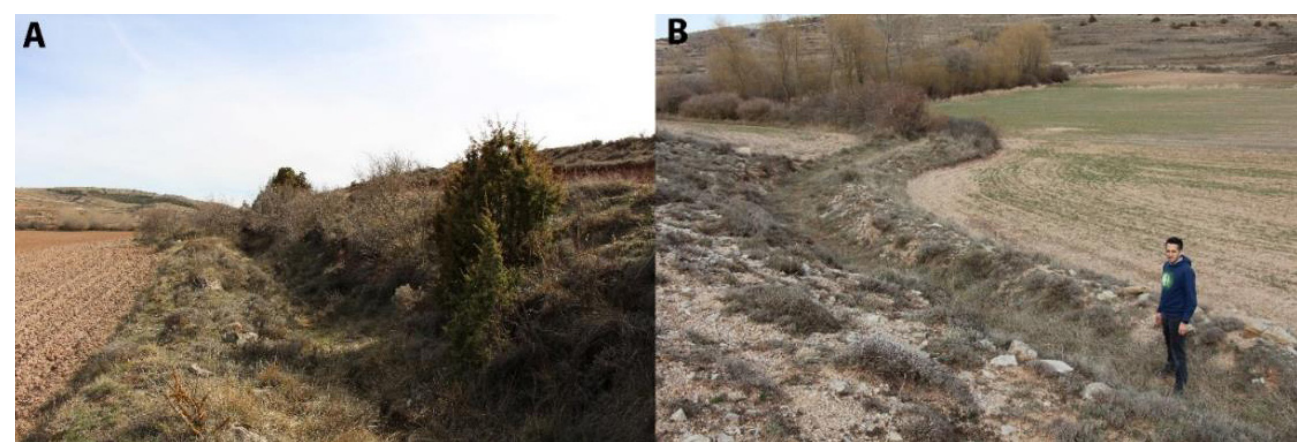

Figura 7A y 7B. Ejemplo de acequia recolectora a pie de ladera (A). Ejemplo de acequia evacuadora (B).

Fotografía de los Autores.

Una técnica de labranza utilizada para controlar la conectividad en los bancales es diseñarlos con pendientes hacia el interior: se hace una zanja en el interior y se conecta con una acequia evacuadora.

\subsubsection{Corrección de cauces}

La lucha contra la erosión se complementa con la modificación del drenaje natural. La finalidad principal de estas acciones es facilitar el drenaje en los campos de cereal: se evita el encharcamiento y se controla la incisión de la red fluvial. Este tipo de actuaciones están presentes en cualquier sector de las cuencas analizadas (figuras.5, 8 y 9).

La corrección de cauces combina desplazamientos de la red, diseño de trazados rectos y excavación de zanjas de drenaje.

a) El desplazamiento de la red a un lateral busca generalmente poder cultivar el centro de una vaguada. Se aplica sobre todo cuando son estrechas. (por ejemplo, barranco del Mas de Ramos, figura 8). Otro objetivo es convertir una vaguada muy estrecha en una cañada de ganado (por ejemplo, Bco. de la Cañadilla, figura 9). En estos casos los cursos se adaptan al trazado irregular de las vaguadas.

b) Los trazados rectos se utilizan en contextos de vaguadas amplias, con poca pendiente y proclives al encharcamiento. Suelen ubicarse en el eje más bajo de la vaguada, y no importa que el terrazgo quede partido en dos. El tramo medio del Barranco de San Cristóbal tiene cauces de este tipo con varios centenares de metros de longitud (figura 8). 


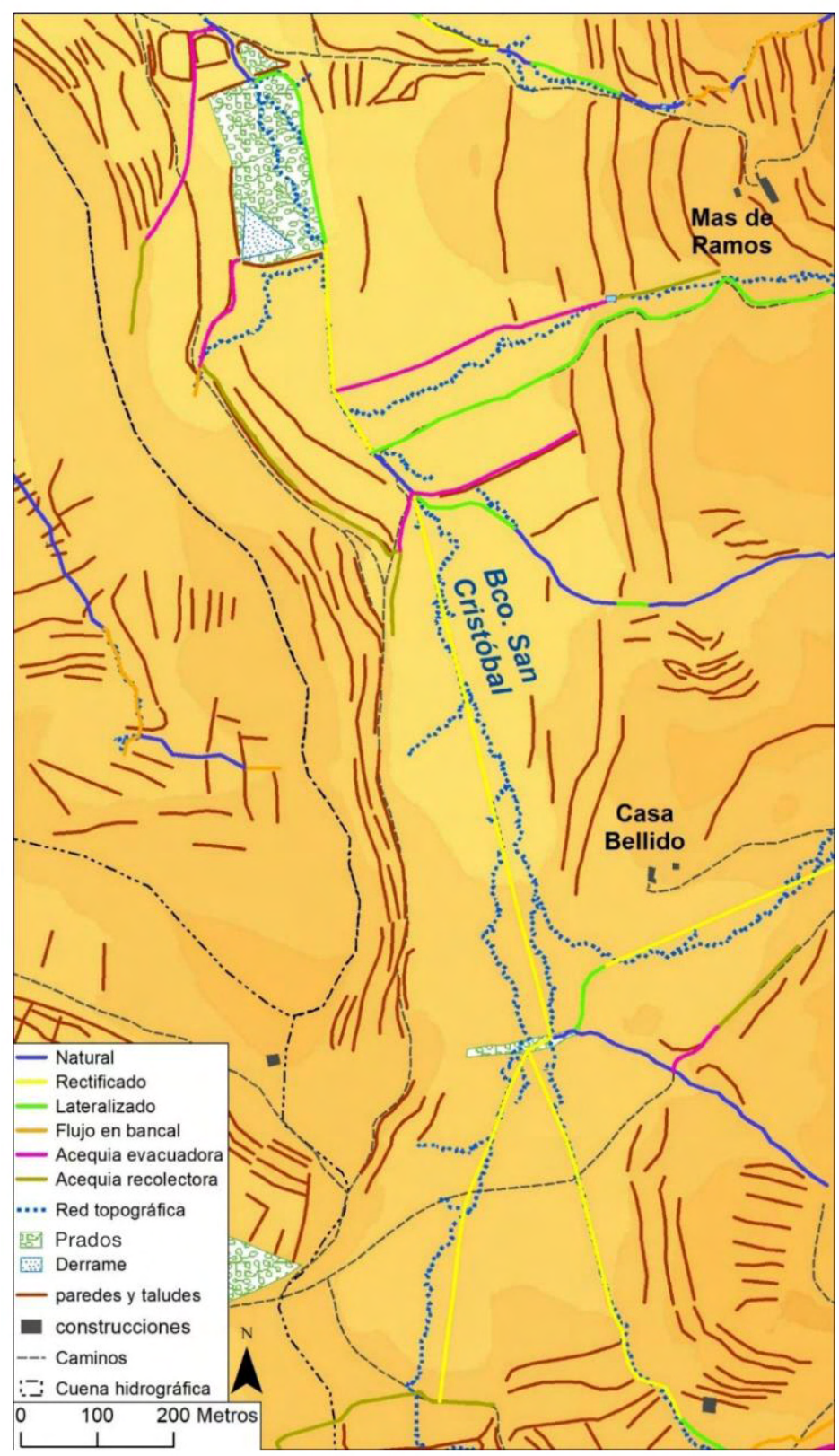

Figura 8. Red de drenaje, elementos de defensa contra la erosión y aprovechamiento de aguas de escorrentía en la parte media del Bco. de San Cristóbal. 
c) La excavación de zanjas supone la aparición de elementos de drenaje totalmente nuevos. Es una solución menos común que las anteriores y puede tener una doble función: es un elemento más de drenaje que refuerza la conectividad del sistema, y puede servir para regar los prados o campos de cereal adyacentes. Para ello, se ubican en zonas llanas bajas y se recrecen los laterales cada vez que se limpian, pues por su propio diseño y ubicación tienden a colmatarse. Al circular el agua a cota más alta que la superficie lateral, pueden derramar el agua en ellos si interesa (ej. la derivación N del tramo final del Bco. de la Cañadilla, figuras 9 y 10).

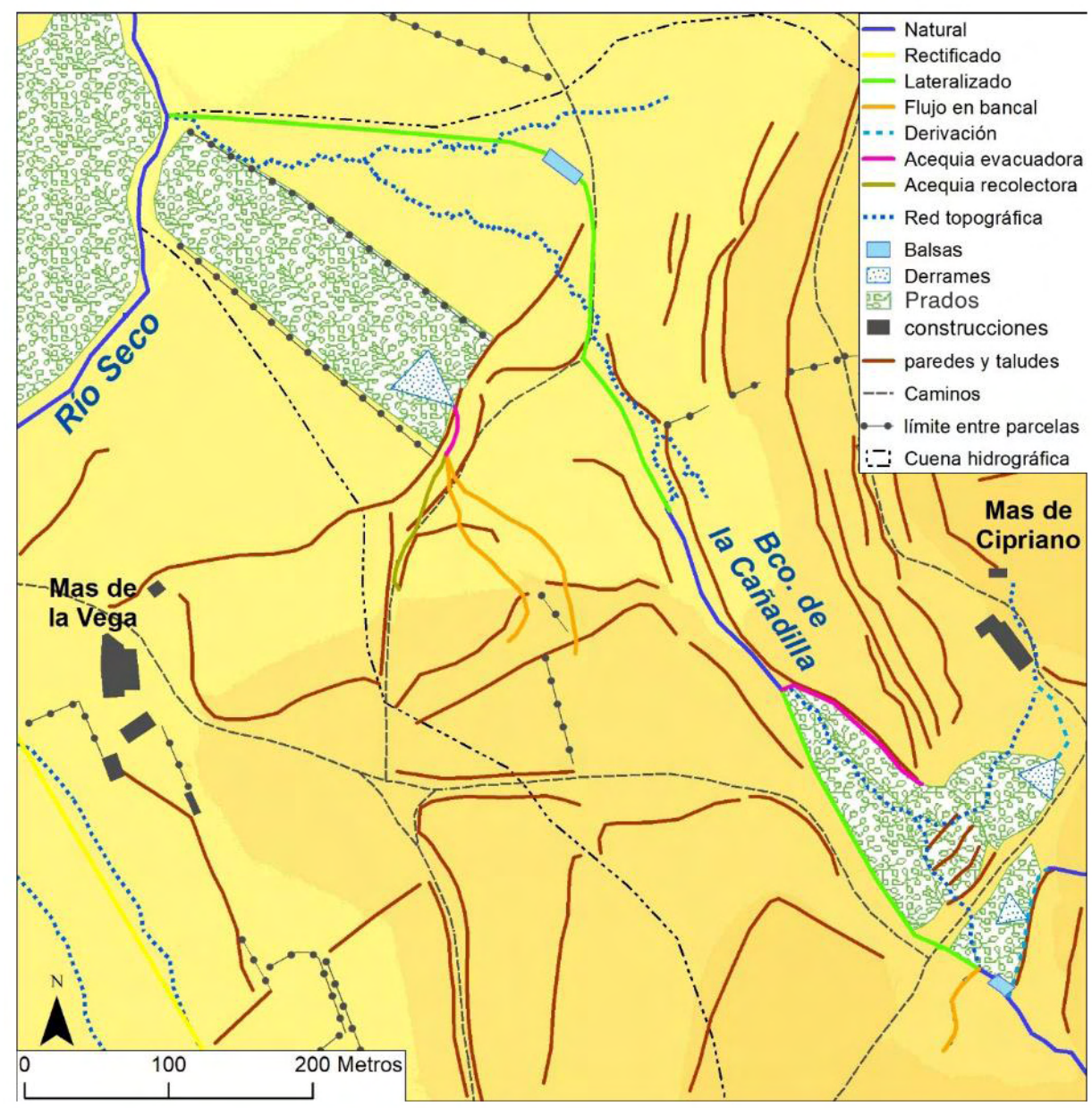

Figura 9. Red de drenaje, elementos de defensa contra la erosión y aprovechamiento de aguas de escorrentía en el tramo bajo del Bco. de la Cañadilla. 


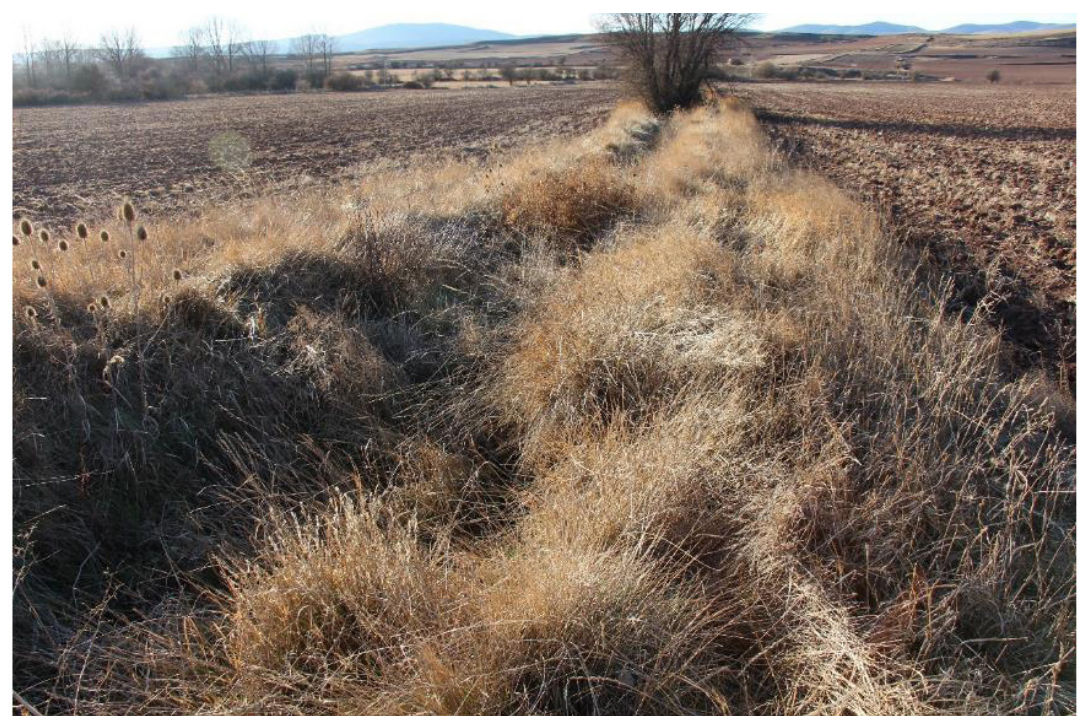

Figura 10. Ejemplo de zanja/acequia que puede ser utilizada para regar.

Fotografía de los Autores.

Esta modalidad de modificación de cauces está a caballo entre el control de la erosión y el aprovechamiento de escorrentías.

En los tres tipos de corrección de la red fluvial natural, un elemento fundamental de control de la erosión es mantener vegetada la acequia, un equilibrio entre una vegetación abundante y bien enraizada, pero que no llegue a taponar el canal.

\subsection{Aprovechamiento de escorrentías}

\subsubsection{Derrames}

Los derrames en prados son el aprovechamiento esencial de la escorrentía. Ya hemos apuntado anteriormente el frágil equilibrio que se da en este sector entre las condiciones climáticas y su dedicación agropecuaria ovina y bovina. Dado que las lluvias invernales son escasas y las tormentas suponen un input hídrico imprescindible cuando hace más calor, para obtener prados más feraces es conveniente regarlos suplementariamente con aguas de arroyada. Si el diseño es adecuado, se convierte en un riego pasivo muy eficiente. Pero, como sólo pueden habilitarse reductos limitados y sin conexión espacial, es necesario cerrarlos con muretes de piedra, para que el ganado no paste libremente. La "cerrada" aislada con prado es un elemento característico del paisaje monteagudino (figura 11). 


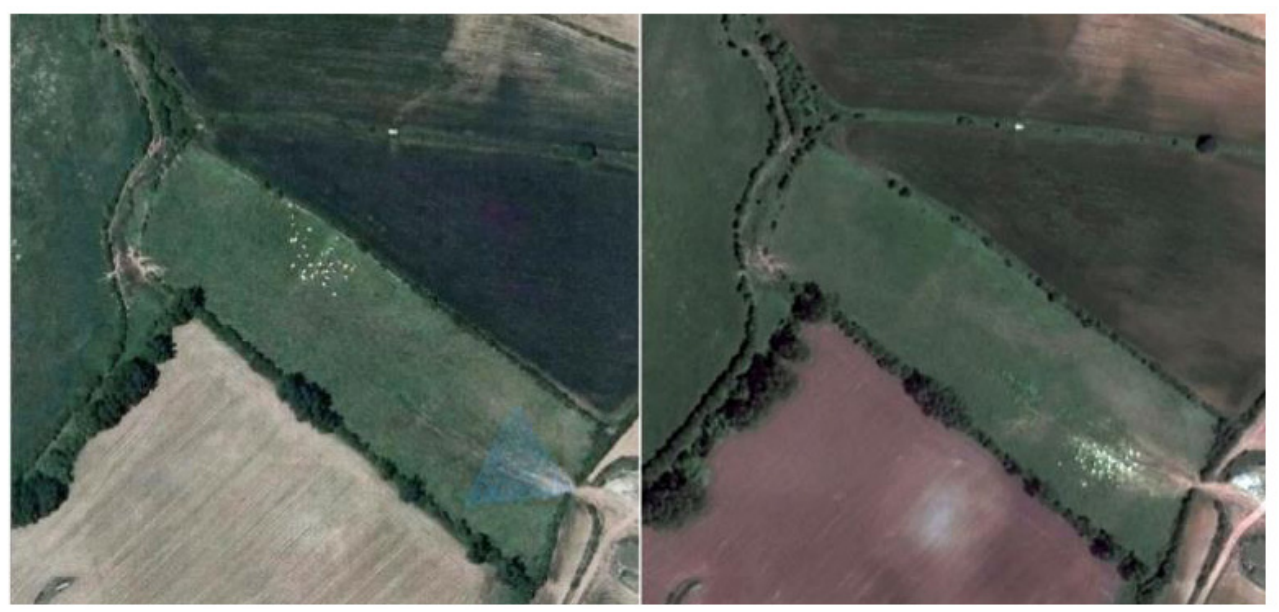

Figura 11. Cerrada con prado para el ganado con derrame de los sobrantes de bancales.

Fuente: A) IGN, 2015. B): Google Earth, 4/2015).

En las figuras 8 y 9 se pueden observar varios ejemplos de prados. La mayor parte de ellos están cerca de colectores importantes de la red fluvial. Para conseguir derramar el agua dentro del prado es necesario realizar siempre algún tipo de modificación de la red. La más fácil es la "laterización" del cauce principal, que va ganando altura respecto a su vaguada, convertida en prado (prado SW del Mas de Cipriano, figura 9). Pero también pueden diseñarse acequias de derivación desde el cauce principal (prados NE del Mas de Cipriano, figura 9), o pueden excavarse acequias de drenaje que queden más altas que las tierras de alrededor. Incluso, puede derramarse en puntos en los que se pierde la red fluvial (derrame final del Bco. de la Cañadilla, figura 9).

Es tan importante este tipo de gestión que nos induce a pensar que el mismo emplazamiento de las masías depende en buena medida de que puedan tener este tipo de elementos: una fuente cercana, un curso de cierta importancia y una topografía del valle mínimamente adecuada en anchura y pendiente. Se consigue tener lo más cerca de la vivienda las cerradas para el ganado y las acequias que tienen que gestionar para obtener derrames en casos de avenida. El mejor ejemplo dentro del sector estudiado es el Mas de Cipriano (figura 12), que tiene incluso una balsa de agua de escorrentía. La cercana Masía de la Vega, situada al final de cuenca adyacente del Bco. del Pilar, también tiene todos estos elementos. 


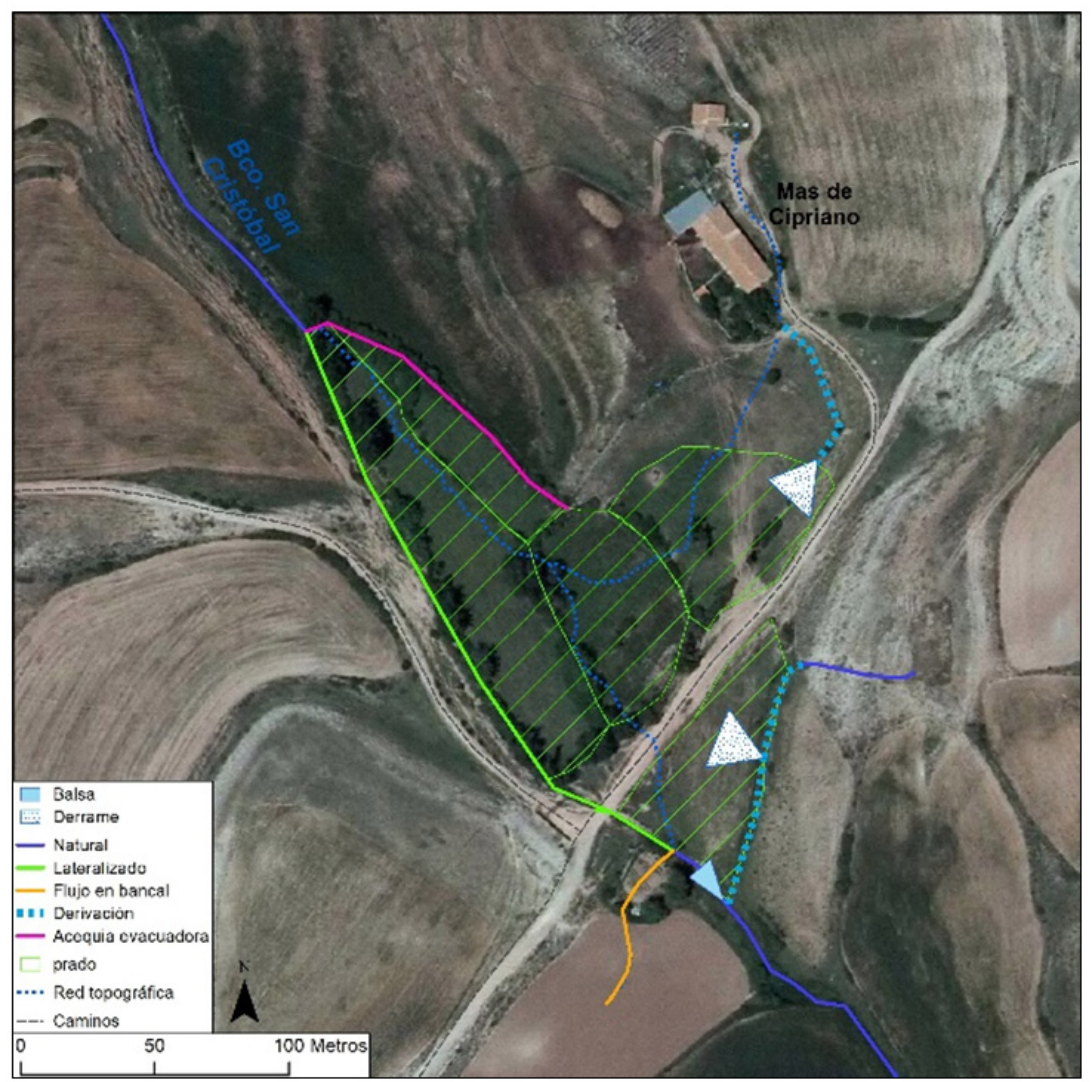

Figura 12. Ejemplo de prado situado aguas abajo de cauce "lateralizado". Fuente: Elaboración propia.

\subsubsection{Balsas}

Es otro tipo de aprovechamiento del agua de escorrentía, aunque menos frecuente. Se trata de balsas asociadas casi siempre a cauces modificados (cauces lateralizados, acequias de derivación) (figuras 9 y 13). Su función es complementar el aprovisionamiento hídrico para el ganado, pero a veces estaban ligadas a microrregadíos de masías, sobre todo si disponían del aporte extra de un pequeño manantial. 


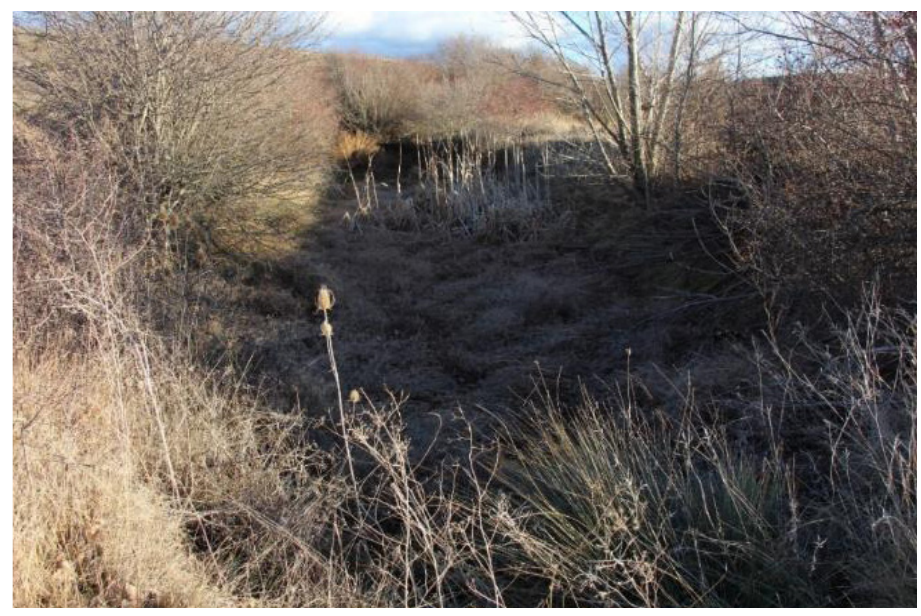

Figura 13. Ejemplo de balsa asociada a una acequia de derivación de escorrentía.

Fotografía de los Autores.

\subsubsection{Caños}

Son zanjas subterráneas rellenas de piedras gruesas y tapadas en superficie con tierra que pudiera ser labrada sin dificultad. Es un sistema de drenaje que se complementa con los "enjuagadores" y acequias de evacuación superficiales, pues permite que el campo no se encharque demasiado sin necesidad de echar mano de estos elementos que fragmentan el espacio cultivable. Es decir, si el encharcamiento es considerable, se echa mano de canales superficiales, pero si es moderado, aunque indeseable, se puede solucionar con caños.

El caño es otro elemento con una doble función, de drenaje y aprovechamiento: a veces, a la salida del caño puede haber una balsa, un pequeño henar, o incluso una fuente. En Monteagudo existen al menos siete fuentes asociadas a caños (Penalba y Pérez Cueva, 2013).

\section{Discusión y conclusiones}

En la gestión de la escorrentía producida por tormentas estivales en Monteagudo del Castillo se observa un delicado equilibrio de adaptación al medio. Esta adaptación se produce tanto en los planos topográfico y litológico, como en el climático: por el potencial riesgo de erosión de un roquedo poco consistente con pendientes elevadas, 
y por la utilización como recurso hídrico de precipitaciones de elevada intensidad. Con ello, se obtienen unos usos del suelo de tipo agropecuario, cerealistas y de ganado bovino, y a la vez se controla la erosión. Se trata, además, de una gestión del agua como riesgo y/o recurso con rápidos cambios en el espacio.

Este sistema de gestión de la escorrentía de tormentas estivales se encuadra, en general, dentro del aprovechamiento de escorrentías en laderas, en el que se distinguen dos elementos claramente diferenciados, el impluvium, o zona de captación de agua, y los elementos de distribución. En este sentido, se asemeja al sistema meskat, que se estructura claramente de este modo (El Amami, 1977), incluso en su esencia de gestión como riesgo/recurso, pues son sistemas que necesitan de lluvias intensas (riesgo) e intentan aprovechar todo el recurso sin generar erosión (Fourati, Lahmar, Khlifi, y Majdoub, 2016).

En el caso estudiado, no obstante, se observan también diseños en los que la escorrentía generada en las laderas discurre concentrada en cauces de valle más o menos antropizados. En todo caso, el área de aprovechamiento (los prados) no siempre están contiguos al área de impluvium (como ocurre siempre en el meskat). Esto asemeja estos sistemas a las "boqueras", aunque no se trataría del concepto clásico (cuencas de cierto tamaño, grandes concentraciones de escorrentía en cauces, aprovechamiento parcial del agua...) (Morales, 1968). Se asemejaría más al concepto de "boqueras de ladera" (Morote, 2013), en los que se produce escorrentía concentrada, pero se aprovecha normalmente al pie de la ladera. Una diferencia sustancial entre estos sistemas y los de meskat es que en este último la escorrentía obtenida en el impluvium es un "recurso generado", mientras que en las boqueras de ladera es un "excedente aprovechable".

En los sistemas de Monteagudo del Castillo se está a caballo entre estas dos concepciones del aprovechamiento de la escorrentía en laderas, aunque predomina la de excedente aprovechable. La originalidad de estos sistemas, que pueden observarse con diseños esencialmente semejantes en otros lugares del Alto Alfambra, radica en el tipo de aprovechamiento del agua, como riego complementario de prados. Se trata de una original adaptación a un rasgo climático singular de este territorio, las tormentas violentas veraniegas. La gestión de esta escorrentía es la clave de la existencia de ganadería bovina con una pluviometría tan escasa, entre 400 y $500 \mathrm{~mm}$.

\section{Bibliografía}

Arnáez, J., Lana-Renault, N., Lasanta, T., Ruiz-Flaño, P. y Castroviejo, J. (2015). Effects of farming terraces on hydrological and geomorphological processes. Catena 128, pp. 122-134.

Beckers, B., Berking, J. y Schütt, B. (2013). Ancient water harvesting methods in the drylands of the Mediterranean and Western Asia. Journal for Ancient Studies 2, pp. 145-164. 
Bracken, L.J. y Croke, J. (2007). The concept of hydrological connectivity and its contribution to understanding runoff dominated geomorphic systems. Hydrological processes, 21(13), pp. $1749-1763$.

Cuadrat, J.M., Saz, M.A. y Vicente-Serrano, S.M. (2007). Atlas Climático de Aragón. Gobierno de Aragón. Zaragoza.

El Amami, S. (1977): The use of runoff waters for agriculture in tunisian arid zones. Study case of water management of meskats in the Sahel of Sousse (Tunisia). Cahiers $d u$ CRGR, Tunisia, 7.

El Amami, S. (1983). Changing conceps of water managements in Tunisia. Impact of Science on Society, 1, pp. 57-64.

Fourati, M., Lahmar, R., Khlifi, S. y Majdoub, R. (2016). Inventaire et diagnostic des aménagements antiérosifs dans le bassin versant d'Oued laya el hammam. Journées scientifiques de la Medjerda, 9-10 Novembre 2016.

González, J. (2006). Climatología de tormentas en España. Meteored y Divulgameteo, 57 pp.

Hernández, M., y Morales, A. (2013). Los aprovechamientos tradicionales de las aguas de turbias en los piedemontes del Sureste de la Península Ibérica: estado actual en tierras alicantinas. Boletín de la Asociación de Geógrafos Españoles, 63, pp. 105-123. DOI: https://doi.org/10.21138/bage.1608

IGME (1981). Mapa geológico de Alcalá de la Selva, escala 1:50.000, serie MAGNA. Instituto Geológico y Minero de España, Servicio de Publicaciones, Ministerio de Industria.

Lozano, M.V. (1983). Estudio geomorfológico de la depresión El Pobo-Cedrillas (Provincia de Teruel). Teruel: Revista del Instituto de Estudios Turolenses, 69, pp. 109-210.

Meerkerk, A.L., Van Wesemael, B. y Bellin, N. (2009). Application of connectivity theory to model the impact of terrace failure on runoff in semi arid catchments. Hydrological Processes, 23(19), pp. 2792-2803. DOI: http://dx.doi.org/10.1002/hyp.7376

Morales Gil, A. (1968). El riego con aguas de avenida en las laderas subáridas. Papeles de Geografia, pp. 167-183.

Morell, J. (2001). El factor de la precipitación en la formación de avenidas en la cuenca alta del Turia. Geographicalia, (40), pp. 47-74.

Morote Seguido, A.F. (2013). El aprovechamiento de turbias en San Vicente del Raspeig (Alicante) como ejemplo de sistema de riego tradicional y sostenible. 59, pp. 147-169. DOI: 10.14198/INGEO2013.59.09

Pailhé, P. (1971). Caractères morphologiques de la dépression d El Pobo (Monts Celtibériques orientaux), Revue géographique des Pyrénées et du Sud-Ouest, Toulouse, 42(1), pp. 71-82.

Pailhé, P. (1984). La chaîne iberique orientale. Étude geomorphologique. Tesis Doctoral, Universidad de Burdeos, III, 682 pp.

Penalba Guillén, J.L. y Pérez-Cueva, A.J. (2013). Las fuentes y manantiales de Monteagudo del Castillo. Valencia, Ed. Repro-Expres, 248 pp.

Penalba Guillén, J.L. y Pérez-Cueva, A.J. y Fansa, G. (2012). Las fuentes de Monteagudo del Castillo (Teruel): Aspectos Hidrogeológicos y características fisicoquímicas de las aguas. Revista del Instituto de Estudios Turolenses. Ed. Instituto de Estudios Turolenses, de la Excelentísima Diputación Provincial de Teruel, 94(I), pp. 131-172. 
Simón_Porcar, G. (2017). Estudio estratigráfico y estructural de los sedimentos neógenos al Este de la Sierra del Pobo (Cordillera Ibérica). Trabajo de fin de grado, Liesa, C. (Dir.), Dep Ciencias de la Tierra, Fac. Ciencia, Univ. Zaragoza, 25 pp., inédito.

Villarroya Gil, F., Pérez-Cueva, A.J., Fansa, G. y Penalba Guillén, J.L. (2014). La hidrogeología sutil: aplicación a la cuenca alta del río Alfambra (Teruel), pp. 887-898. En: Gómez, J.J. y Rodrigo, J. (Eds.), II Congreso Ibérico de las Aguas Subterráneas. Libro de Actas. CIAS2014, Valencia, del 8 al 10 de septiembre de 2014, 909 pp. 